\title{
Noise Removal and Blob Identification Approach for Number Plate Recognition
}

\author{
Reshma P. \\ Dept. of Computer Science \\ Tushar Patnaik \\ Sr. Engineer and Lecturer \\ Centre for Development of Advanced Computing(C-DAC), Noida
}

\begin{abstract}
Noise removal method is used to pre process the input image and blob detection method is used to localize and segment Indian number plates. The segmented characters on the number plate region are compared with the previously stored character database and finally approve authentication or access permission for different vehicles.
\end{abstract}

\section{Keywords}

Noise components, blobs, Blob identification, morphological operation, aspect ratio, classification

\section{INTRODUCTION}

Automatic Vehicle Identification is a complex at the same time an actual problem of our current scenario. AVIS has complex characteristics due to diverse effects. The developed method is a fast, real time useable solution for this problem. The work concentrates on different issues like skewed images, unaligned characters in the number plate, different resolution and different illumination conditions. The work also contains an implementation of a demonstration model along with screenshots which would be further help in preceding these functions successfully.

The proposed system has many applications in traffic surveillance system such as automatic toll collection centers Parking lots and petrol stations. Other applications include journey time monitoring, red light violation enforcement etc. In this study, a smart and simple algorithm is presented for localization and segmentation of number plate characters.

\section{EXISTING SYSTEM}

Indian number plates can have single or double row. A number of algorithms are proposed and implemented for number plate localization such as multiple interlacing algorithms, transform domain filtering, image filtering and smearing algorithm and color image processing. For character segmentation, row and column segmentation and image scissoring algorithm are used. The different approaches generally used for character recognition optical character recognition, artificial intelligence and statistical feature extraction.

\section{SYSTEM STRUCTURE}

The proposed approach divides the whole system into five modules. First module deals with image acquisition and preprocessing of input images. The second module concentrates on detection of number plate region from the different identified blobs. A classification algorithm is used to differentiate the other blobs from license plate region. The third module is all about segmentation of each alpha numeric characters present in the localized number plate region and fourth module proceeds towards recognizing the number plate. Finally a validation module is incorporated to provide authentication of identified vehicles.

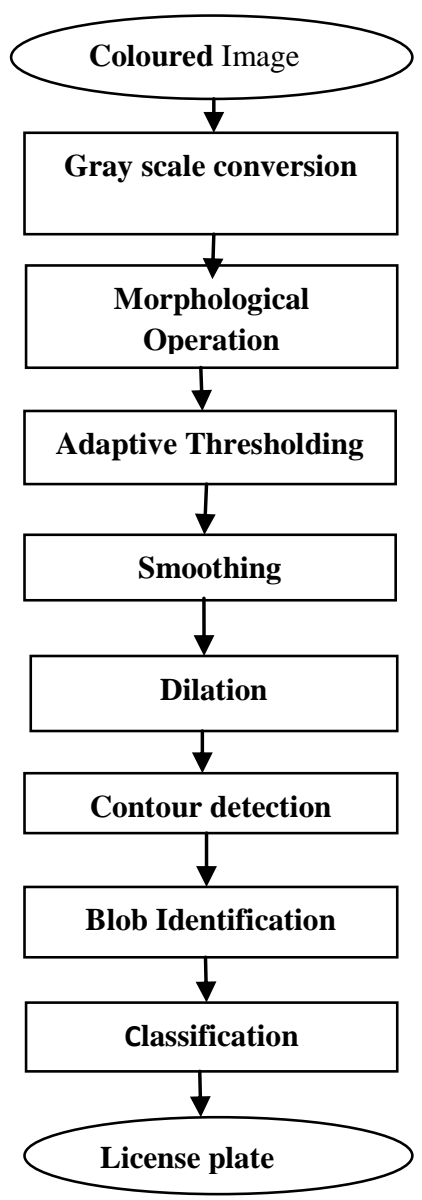

Fig 1: Flow Chart of Localization 


\section{TOOLS USED}

OpenCV with Visual $\mathrm{C}++$ is the best combination for computer vision. OpenCV is a library of programming functions for real time computer vision and it is free for use under the open source BSD. OpenCV provides interface for different camera models. Visual Studio 2008 enables organizations of every size to rapidly create more secure, manageable, and reliable applications that take advantage of Windows Vista ${ }^{\mathrm{TM}}$ and the 2007 Office system. Visual Studio 2008 delivers key advances for developers in three primary pillars: Rapid application development, Effective team collaboration and Breakthrough user experiences.

\section{IMPLEMENTATION DETAILS}

\subsection{Image Acquisition}

The image of the vehicle is captured using a photographic camera. The constraint is that the image of the vehicle should be captured in such a way that the selected input image contains rear or front view of the vehicle with the number plate. First the size of the image is resized to a feasible aspect ratio. Then the coloured image is converted into gray scale image. The following code snippet explains how to resize an image in OpenCV.

cvCreateImage(cvSize(img-> width/10,img->height/ 10), img>depth,img->nchannels);

\subsection{Preprocessing}

The resized image is given to further processing.

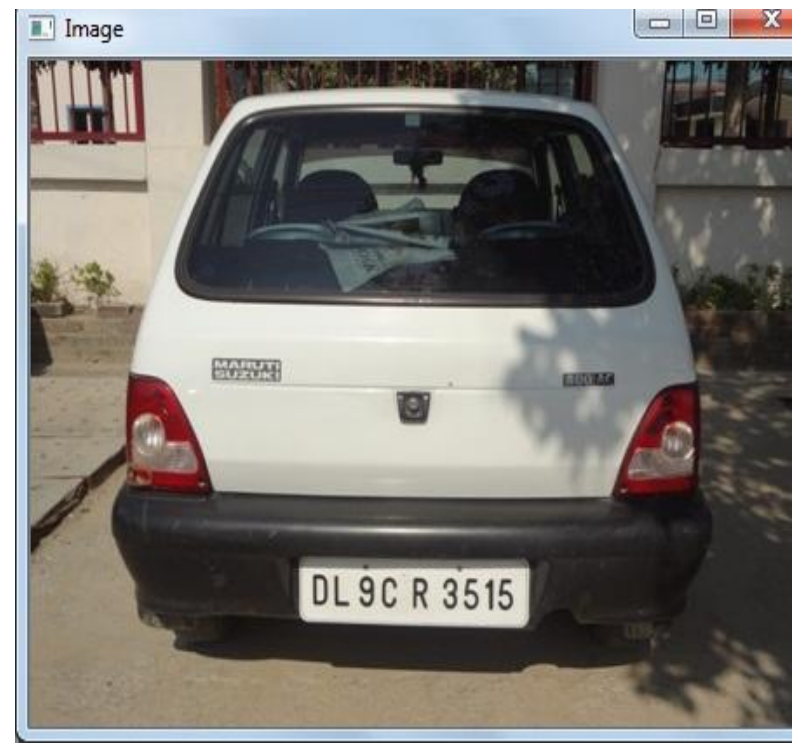

Fig 2: Input Image

Preprocessing of the given coloured image involves many stages. The above coloured image is first converted in to gray scale image. Then one of the morphological operations called Top Hat is applied. Morphological Top Hat operation is used to remove noise and extract details.

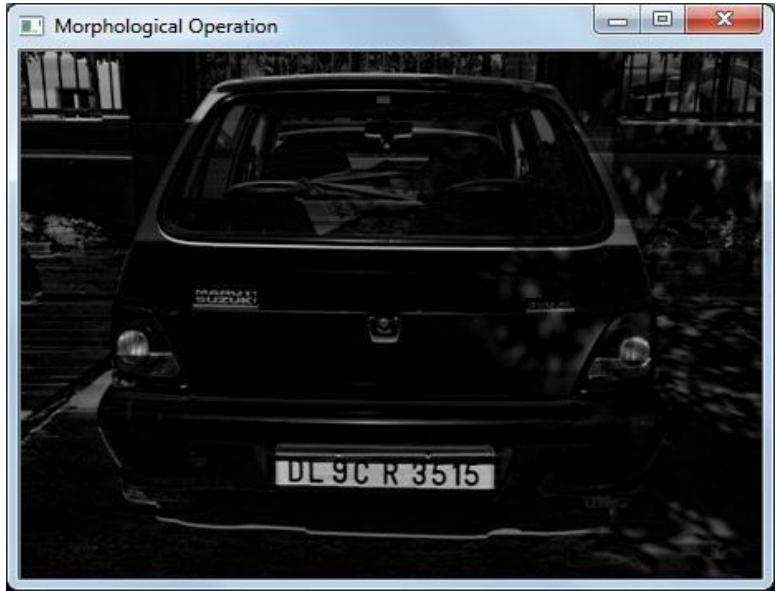

Fig 3: Output of Top Hat Operation

Then the output of Top Hat is thresholded between the values 128 and 255 and CV_THRESH_BINARY method is applied.

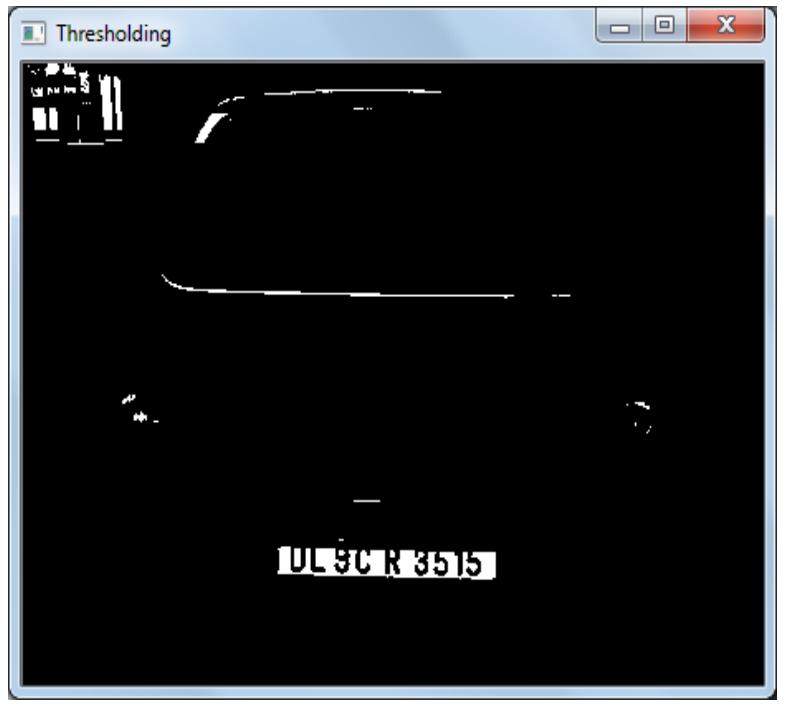

Fig 4: Output of Thresholding

Thresholding is used for highlighting characters and suppressing background. CV_GAUSSIAN method is used to blur images and further used in order to remove the noise components. Dilation procedure is applied which uses a structuring element for expanding the shapes contained in the input images.

Threshold is an image processing operation by which the pixels of the image are truncated to two values depending upon the value of threshold. This operation is used to convert the image to binary and localize the license plate from the image of the vehicle. Thresholding requires pre image analysis for identifying the suitable threshold value. Many statistical and physical modeling algorithms have been developed for the same purpose. An adaptive thresholding technique called Otsu's method is used for binarisation.

Applies fixed-level threshold to array elements:-void cvThreshold( const CvArr* src, CvArr* dst, double threshold, double maxValue, int thresholdType ); 


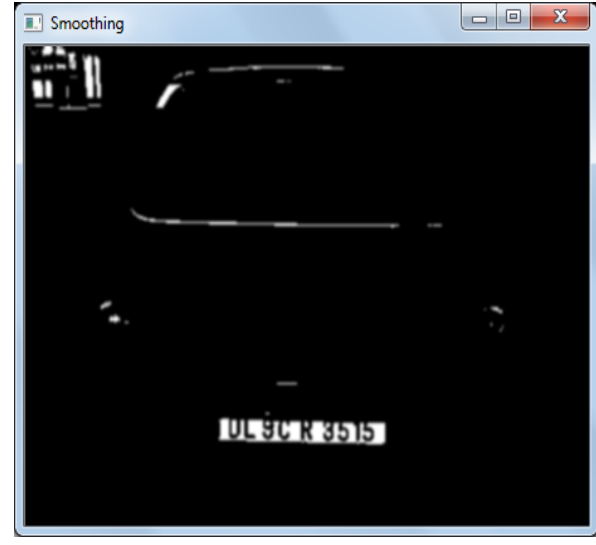

Fig 5: Output of Smoothing

Dilation procedure is applied which uses a structuring element for expanding the shapes contained in the input images.

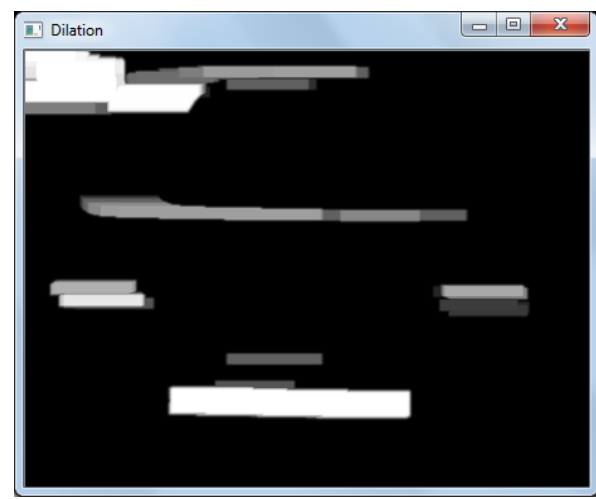

Fig 6: Output of Dilation

\subsection{Localization}

Localization refers to the extraction of needed area from the vehicle image. The area interests us is number plate region and hence the number plate and some part of vehicle is obtained by the extraction technique. The output of last pre processing stage that is dilated output is used as the input for localization of number plate from the given image. First find the contours using the method cvFindContour and the corresponding output is given below.

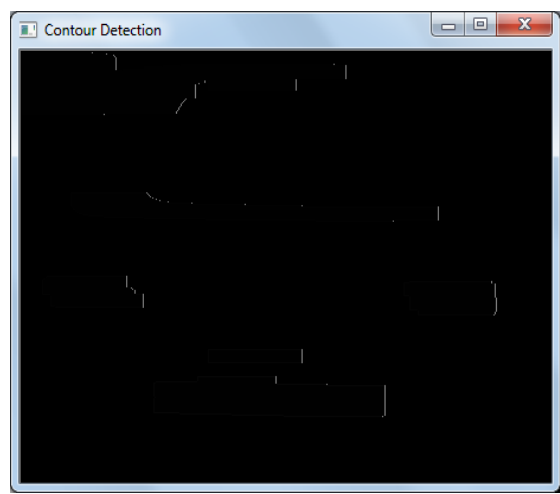

Fig 7: Contour Detection

The output contours are given to a blob identification module in which the same intensity components are being identified and marked as blobs.

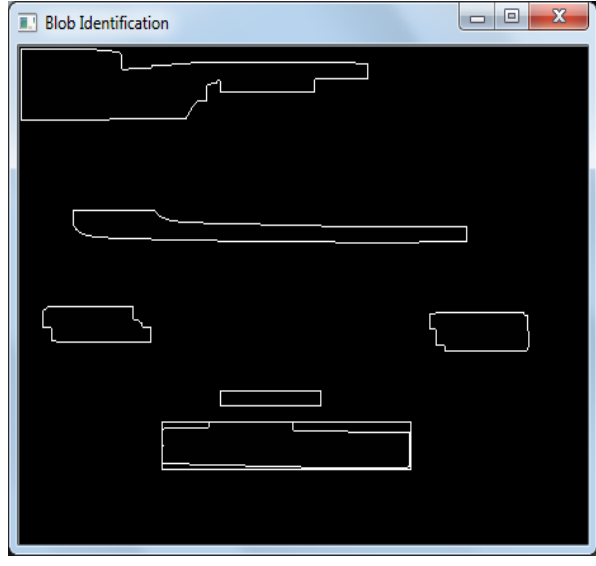

Fig 8: Output of Blob identification module

In the blob identification module, many blobs having the same intensities are identified and among them only the number plate region is need to be extracted. For that a classification algorithm is used. The algorithm defines an aspect ratio based elimination method which helps in extracting only the needed number plate region from the blobs being identified. All other blobs are being discarded.

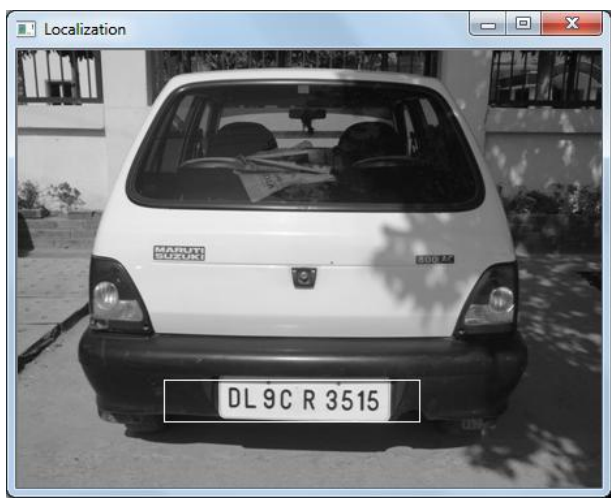

Fig 9: Output of Classification

The classification algorithm works according to aspect ratio which calculates the width and height ratio of each blobs and only the blobs having feasible value of aspect ratio are extracted after number plate localization.

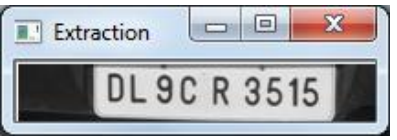

Fig 10: Output of extracted Number plate

\subsection{Segmentation}

The localized output having extracted number plate region is given to the segmentation module for separating each alpha numeric character. The above output is segmented using connected component analysis and bounding box is generated around each character present in the number plate region. 

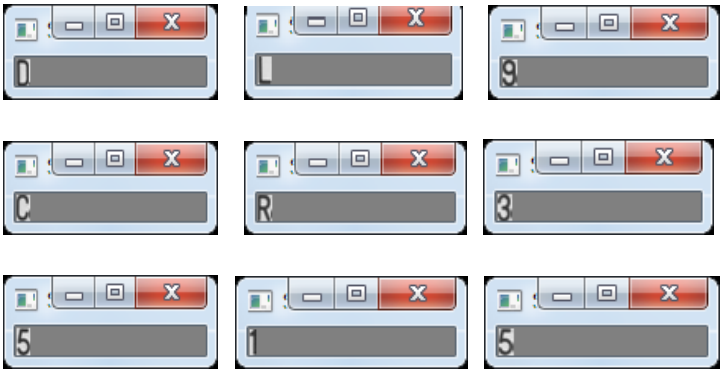

Fig 11: Segmented Number Plate

Now, all the segmented characters are extracted and smoothing operation is applied in order to eliminate noise components. Then each character is saved as individual character images.

From the number plate region each and every alpha numeric characters are extracted using bounding box principle and only characters and numbers in the number plate region are extracted. These segmented characters are the input to recognition module for further identifying the number plate.

The segmented characters obtained are given to recognition module. Here, template matching is used to identify each alpha numeric characters present in the number plate. Each segmented character is compared with the previously stored characters present in the template database which is called character database. Whenever there is a matching occur between segmented character and character database then that character is identified and displayed. Then check for next character and so on. Finally, the valid number plate is getting identified and displayed in the command prompt or graphical user interface.

\subsection{Character Recognition}

Template matching is one of the most common and easy classification methods for recognizing the characters.

\section{ABCDEFGHIJKL}

MNOPRSTUVYZ

\section{9}

Fig. 12: Template Database

Template matching and statistical feature extraction is used for the recognition of each alpha numeric characters. For that a character database is maintained with 0 to 9 numbers and A to $\mathrm{Z}$ English alphabets. The size of each character image is normalized according to the size of characters stored in the database. Here each character is fit to $30 \mathrm{x} 50$ and is compared for template matching.

In template matching module the number plate is segmented and the images containing individual characters forming the number plate are obtained. Each normalized character and number present in the number plate is compared with the alpha numeric characters present in the previously stored character database. License plate is compared to its corresponding pixel in the template.

\subsection{Validation}

In the validation module, if the characters present in the number plate and characters present in the character database are matching then validation is successful and authorization is given to that particular vehicle for security and automated applications. This system can be used for automation of petrol stations, automatic parking lots, journey time analysis etc.

\section{CONCLUSION}

The system works satisfactorily for wide variations in illumination conditions and different types of number plates such as tilted, italic and bold characters found in India. The proposed method is suitable for real time conditions. The major sources of error were the non English script and extreme variations in the dimensions of number plate characters.

\section{FUTURE SCOPE}

The system can be redesigned for multinational license plates and images having more than one number plate.

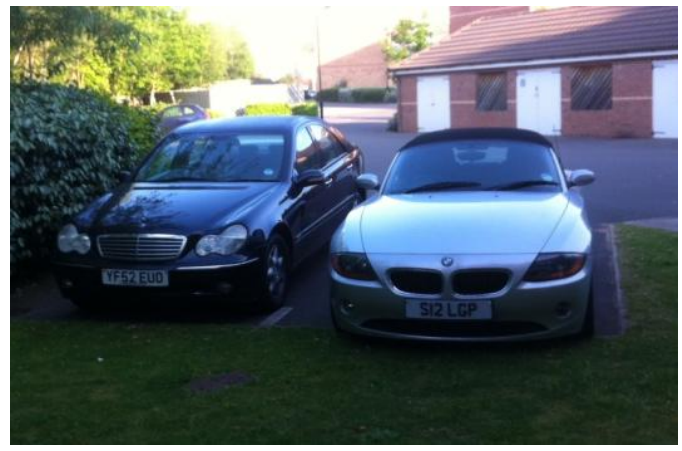

Fig. 12

\section{ACKNOWLEDGEMENT}

Bunch of thanks to my project guide Mr. Tushar Patnaik, and the whole OCR team at CDAC, Noida, for mentoring this research. Next, I would like to mention my sincere thanks to my husband, Mr. Anukiran R, my only daughter Bhadra Kiran Menon, my parents and in-laws for their kind support and patient understanding. 


\section{REFERENCES}

[1] Stokman, Harro and Gevers, Theo. "Selection and Fusion of Color Models for Image Feature Detection." IEEE Transactions on Pattern Analysis and Machine Intelligence 29.3 (2007): 371-381.

[2] Vahid Abolghasemi, Alireza Ahmadyfard. "An edgebased color-aided method for license plate detection." Image and Vision Computing, Volume 27, Issue 8, 2 July 2009, pp. 1134-1142.

[3] Prateek Banga Automatic Number Plate RecognitionSystem for Indian conditions" 978-1-42443538-8/09/\$25.00 @2009 IEEE

[4] Parking surveillance and number plate recognition application Pletl Szilveszter, Gálfi Csongor SISY 2010 • 2010 IEEE 8th International Symposium on Intelligent Systems and Informatics September 10-11, 2010, Subotica, Serbia

[5] A Feature Based Approach for Localization of Indian Number Plates Prathamesh Kulkarni , Ashish Khatri, Prateek Banga, Kushal Shah 978-1-4244-33551/09/\$25.00@2009 IEEE

[6] Automatic Number Plate Recognition (ANPR) System for Indian Conditions using Support Vector Machine (SVM) by Shriram Kishanrao Waghmare, A. K. Gulve,
Vikas N.Nirgude and Nagraj P. Kamble International Journal of Computer Science and its Applications

[7] Automatic Vehicle Identification by Plate RecognitionSerkan Ozbay, and Ergun Ercelebi -World Academy of Science, Engineering and Technology 92005

[8] Contour and Texture Analysis for Image SegmentationJitendra Malik, Serge Belongie, Thomas Leung*and Jianbo Shi-International Journal of Computer Vision 43(1), 7-27, 2001c 2001 Kluwer Academic Publishers.Manufactured in The Netherlands.

[9] Automatic License Plate Localization Using Intrinsic Rules Saliency Chirag N. Paunwala , Dr. Suprava Patnaik (IJACSA), Special Issue on Image Processing and Analysis

\section{AUTHOR'S PROFILE}

Ms Reshma P, completed M.Tech in Computer Science from C DAC, NOIDA, B.Tech in Information Technology from MES College of Engineering, Kuttippuram, and works as an Assistant Professor in Dept. of Information Technology at Amal Jyothi College of Engineering, Kerala. 